\title{
TENSOR PRODUCTS AND JOINT NUMERICAL RANGE
}

\author{
A. T. DASH ${ }^{1}$
}

\begin{abstract}
It is shown that the joint numerical range of the tensor product of several operators is the cartesian product of their numerical ranges.
\end{abstract}

Here and in what follows, $\mathscr{H}$ is a complex Hilbert space and by an operator on $\mathscr{H}$ we mean a bounded linear transformation from $\mathscr{H}$ into itself. We denote by $\mathscr{L}(\mathscr{H})$ the algebra of all bounded operators on $\mathscr{H}$. Next we need the following definition in the sequel.

Definition. Let $A=\left(A_{1}, \cdots, A_{n}\right)$ be any $n$-tuple of operators on $\mathscr{H}$. Then we define the joint numerical range of $A$ to be the set $W(A)$ consisting of all $z=\left(z_{1}, \cdots, z_{n}\right)$ of $C^{n}$ (the $n$-dimensional complex space) such that for some $f$ in $\mathscr{H}$ with $\|f\|=1$ we have for each $j, z_{j}=\left\langle A_{j} f, f\right\rangle$; that is,

$$
W(A)=\left\{\langle A f, f\rangle=\left(\left\langle A_{1} f, f\right\rangle, \cdots,\left\langle A_{n} f, f\right\rangle\right):\|f\|=1, f \in \mathscr{H}\right\} .
$$

For further details about joint numerical range the reader is referred to [1]. See also [4].

Let $\mathscr{H}_{1}, \cdots, \mathscr{H}_{n}$ be complex Hilbert spaces. Further, let $I_{j}$ be the identity operator and $A_{j}$ an arbitrary operator on $\mathscr{H}_{j}, 1 \leqq j \leqq n$. We consider the tensor product of operators $T_{j}(1 \leqq j \leqq n)$ acting on the tensor product space $\mathscr{H}_{1} \otimes \cdots \otimes \mathscr{H}_{n}$ defined by

and

$$
\begin{aligned}
& T_{1}=A_{1} \otimes I_{2} \otimes \cdots \otimes I_{n}, \\
& T_{2}=I_{1} \otimes A_{2} \otimes I_{3} \otimes \cdots \otimes I_{n},
\end{aligned}
$$

$$
T_{j}=I_{1} \otimes \cdots \otimes I_{j-1} \otimes A_{j} \otimes I_{j+1} \otimes \cdots \otimes I_{n},
$$

in general. The operators $T_{j}$ obviously commute. For a good account of tensor products of Hilbert spaces and operators, the reader may consult

Presented to the Society, April 3, 1972; received by the editors July 20, 1972.

AMS (MOS) subject classifications (1970). Primary 47A10, 47D20; Secondary 46M05.

Key words and phrases. Algebra of operators, tensor products, convex sets, joint spectrum, joint numerical range, double commutant, commutative Banach algebra, universal property.

${ }^{1}$ This research was partially supported by NRC Grant A07545.

(c) American Mathematical Society 1973 
Dixmier [3]. See also [6]. Let $\mathscr{U}$ be the double commutant of $T_{1}, \cdots, T_{n}$; that is, the set of all operators on $\mathscr{H}_{1} \otimes \cdots \otimes \mathscr{H}_{n}$ which commute with any operator commuting with all of $T_{j} . \mathscr{U}$ is a commutative Banach algebra. A complex vector $z=\left(z_{1}, \cdots, z_{n}\right)$ of $C^{n}$ is in the joint spectrum $\sigma\left(T_{1}, \cdots, T_{n}\right)$ of the operators $T_{j}$ if and only if for all operators $B_{1}, \cdots, B_{n}$ in $\mathscr{U}$

$$
\sum_{j=1}^{n} B_{j}\left(T_{j}-z_{j}\right) \neq I_{1} \otimes \cdots \otimes I_{n}
$$

For facts about joint spectrum see [1], [2] and [4]. It is known that the joint spectrum of the operators $T_{j}(1 \leqq j \leqq n)$ is the cartesian product of their spectra; that is [5]

$$
\sigma\left(T_{1}, \cdots, T_{n}\right)=\prod_{j=1}^{n} \sigma\left(T_{j}\right)=\prod_{j=1}^{n} \sigma\left(A_{j}\right) .
$$

The purpose here is to prove an analogous assertion for joint numerical range which is motivated by the paper of the author and Schechter [5].

Before we state our main result, it may be appropriate to point out that the joint numerical range of an $n$-tuple of operators is not in general convex. Furthermore, it is not known whether or not the joint numerical range of an $n$-tuple of commuting operators is convex. Consult [1] and [4]. However, the joint numerical range of the operators $T_{j}$ is convex which is an immediate consequence of the following theorem.

THEOREM. The joint numerical range of the operators $T_{j}(1 \leqq j \leqq n)$ is the cartesian product of their numerical ranges; that is,

$$
W\left(T_{1}, \cdots, T_{n}\right)=\prod_{j=1}^{n} W\left(T_{j}\right)=\prod_{j=1}^{n} W\left(A_{j}\right) .
$$

Thus it is convex.

We present here several propositions which lead to the proof of the Theorem. To proceed further, we need the following notions and terminologies.

Let $\mathscr{H}$ and $\mathscr{K}$ be any two complex Hilbert spaces. We denote by $\mathscr{H} \odot \mathscr{K}$ their algebraic tensor product (the set of all finite sums $\left.\sum_{j=1}^{n} f_{j} \otimes g_{j}, f_{j} \in \mathscr{H}, g_{j} \in \mathscr{K}\right)$ and $\mathscr{H} \otimes \mathscr{K}$ their Hilbert space tensor product. We recall that $\mathscr{H} \otimes \mathscr{K}$ is the Hilbert space completion (that is, it is the completion of $\mathscr{H} \odot \mathscr{K}$ for a scalar product which satisfies $\left.\left\langle f_{1} \otimes g_{1}, f_{2} \otimes g_{2}\right\rangle=\left\langle f_{1}, f_{2}\right\rangle\left\langle g_{1}, g_{2}\right\rangle\right)$ of $\mathscr{H} \odot \mathscr{K}$. Consult [3] and [6].

Proposition 1. Let $\mathscr{H}$ and $\mathscr{K}$ be any two complex Hilbert spaces and let $E$ be a complex vector space. If $\varphi$ is a bilinear mapping of $\mathscr{H} \times \mathscr{K}$ 
into $E$, then there exists a unique linear mapping $V: \mathscr{H} \odot \mathscr{K} \rightarrow E$ such that $\varphi(f, g)=V(f \otimes g)$.

This is often known as the "universal property". See [6] and [8].

The following proposition is crucial to the proof of the Theorem. This is probably well known. See for instance [8, Exercise 39.1, p. 410]. However, we were unable to find the exact reference. We will give a proof here for the sake of completeness, and for the benefit of the reader.

Proposition 2. Consider the tensor product spaces $\mathscr{H} \otimes \mathscr{K}$ and $\mathscr{K} \otimes \mathscr{H}$. Then there exists a unique bounded linear mapping $U: \mathscr{H} \otimes \mathscr{K} \rightarrow$ $\mathscr{K} \otimes \mathscr{H}$ such that $U(f \otimes g)=g \otimes f$. This mapping is unitary; that is, it is bijective, and $\langle U u, U v\rangle=\langle u, v\rangle$ for all $u, v$ in $\mathscr{H} \otimes \mathscr{K}$.

Proof. We take $E=\mathscr{K} \odot \mathscr{H}$, and define a mapping $\varphi: \mathscr{H} \times \mathscr{K} \rightarrow$ $\mathscr{K} \odot \mathscr{H}$ by $\varphi(f, g)=g \otimes f$. Evidently $\varphi$ is bilinear. Thus by Proposition 1 there exists a unique linear mapping $U: \mathscr{H} \odot \mathscr{K} \rightarrow \mathscr{K} \odot \mathscr{H}$ such that $U(f \otimes g)=g \otimes f$. Clearly, $U$ is surjective. Now we show that $U$ preserves scalar products. Let $u=\sum_{i} f_{i} \otimes g_{i}$ and $v=\sum_{j} f_{j}^{\prime} \otimes g_{j}^{\prime}$ be any two vectors in $\mathscr{H} \odot \mathscr{K}$. Then

$$
\begin{aligned}
\langle U u, U v\rangle & =\left\langle\sum_{i} g_{i} \otimes f_{i}, \sum_{j} g_{j}^{\prime} \otimes f_{j}^{\prime}\right\rangle \\
& =\sum_{i} \sum_{j}\left\langle g_{i} \otimes f_{i}, g_{j}^{\prime} \otimes f_{j}^{\prime}\right\rangle=\sum_{i} \sum_{j}\left\langle g_{i}, g_{j}^{\prime}\right\rangle\left\langle f_{i}, f_{j}^{\prime}\right\rangle \\
& =\sum_{i} \sum_{j}\left\langle f_{i}, f_{j}^{\prime}\right\rangle\left\langle g_{i}, g_{j}^{\prime}\right\rangle=\sum_{i} \sum_{j}\left\langle f_{i} \otimes g_{i}, f_{j}^{\prime} \otimes g_{j}^{\prime}\right\rangle \\
& =\left\langle\sum_{i} f_{i} \otimes g_{i}, \sum_{j} f_{j}^{\prime} \otimes g_{j}^{\prime}\right\rangle \\
& =\langle u, v\rangle .
\end{aligned}
$$

Since $\mathscr{H} \odot \mathscr{K}$ and $\mathscr{K} \odot \mathscr{H}$ are respectively dense in $\mathscr{H} \otimes \mathscr{K}$ and $\mathscr{K} \otimes \mathscr{H}$, there is a unique continuous linear extension which we also denote by $U: \mathscr{H} \otimes \mathscr{K} \rightarrow \mathscr{K} \otimes \mathscr{H}$. Evidently $U$ still preserves scalar products, and hence norm. This implies that its range is complete, and therefore closed. But the range contains $\mathscr{K} \odot \mathscr{H}$, and so must be dense in $\mathscr{K} \otimes \mathscr{H}$. Thus we have $U(\mathscr{H} \otimes \mathscr{K})=\mathscr{K} \otimes \mathscr{H}$.

Proposition 3. Let $A$ be in $\mathscr{L}(\mathscr{H})$ and $B$ be in $\mathscr{L}(\mathscr{K})$, and $U$ be as given in the preceding proposition. Then:

(a) $A \otimes B=U^{*}(B \otimes A) U$, where star represents adjoint.

(b) The numerical range of $A \otimes B$ is the same as that of $B \otimes A$; that is, $W(A \otimes B)=W(B \otimes A)$. 
Proof. (a). We have

$$
\begin{aligned}
U^{*}(B \otimes A) U(f \otimes g) & =U^{*}(B \otimes A)(g \otimes f) \\
& =U^{*}(B g \otimes A f)=A f \otimes B g \\
& =(A \otimes B)(f \otimes g) .
\end{aligned}
$$

They agree on a dense subset, and therefore the result follows. (b). This is an immediate consequence of (a) and the property of $U$.

Proposition 4. Let $\mathscr{H}_{1}, \mathscr{H}_{2}$ and $\mathscr{H}_{3}$ be complex Hilbert spaces, and let $A_{1}$ be in $\mathscr{L}\left(\mathscr{H}_{1}\right)$. Then $W\left(A_{1} \otimes I_{2}\right)=W\left(I_{3} \otimes A_{1}\right)=W\left(A_{1}\right)$.

Proof. First we prove that $W\left(A_{1} \otimes I_{2}\right)=W\left(A_{1}\right)$. If $z$ is in $W\left(A_{1}\right)$, there exists $f$ in $\mathscr{H}_{1}$ with $\|f\|=1$ such that $z=\left\langle A_{1} f, f\right\rangle$. Let $g$ be any unit vector in $\mathscr{H}_{2}$. Set $u=f \otimes g$. Clearly, $u$ is in $\mathscr{H}_{1} \otimes \mathscr{H}_{2}$ with $\|u\|=1$, and

$$
\left\langle\left(A_{1} \otimes I_{2}\right) u, u\right\rangle=\left\langle A_{1} f \otimes g, f \otimes g\right\rangle=\left\langle A_{1} f, f\right\rangle=z .
$$

This implies that $W\left(A_{1}\right) \subset W\left(A_{1} \otimes I_{2}\right)$.

To prove the reverse inclusion, we need the following fact. Let $X$ be a convex subset of $\boldsymbol{C}$. If $\left\{z_{n}\right\}$ is a sequence of elements in $X$ and $\left\{\alpha_{n}\right\}$ is a sequence of numbers such that $\alpha_{n}>0$ and $\sum \alpha_{n}=1$, then $\sum_{n=1}^{\infty} \alpha_{n} z_{n}$ is in $X$. This was recently proved by J. P. Williams. Now let $z$ be any element of $W\left(A_{1} \otimes I_{2}\right)$. Then there is a unit vector $u$ in $\mathscr{H}_{1} \otimes \mathscr{H}_{2}$ such that $z=$ $\left\langle\left(A_{1} \otimes I_{2}\right) u, u\right\rangle$. Next we must show that $z$ is in $W\left(A_{1}\right)$. If $\left\{e_{\alpha}\right\}_{\alpha \in \Lambda}$ is an orthonormal basis for $\mathscr{H}_{2}$, then $u$ can be expressed uniquely as $u=\sum_{\alpha \in \Lambda} f_{\alpha} \otimes e_{\alpha}$ for some family vectors $\left\{f_{\alpha}\right\}_{\alpha \in \Lambda}$ in $\mathscr{H}_{1}$ such that $\|u\|^{2}=1=\sum_{\alpha \in \Lambda}\left\|f_{\alpha}\right\|^{2}$. Consult [3, p. 22]. Thus we have

$$
\begin{aligned}
z & =\left\langle\left(A_{1} \otimes I_{2}\right) u, u\right\rangle \\
& =\left\langle\sum A_{1} f_{\alpha} \otimes e_{\alpha}, \sum f_{\alpha} \otimes e_{\alpha}\right\rangle \\
& =\sum_{\alpha \in \Lambda}\left\langle A_{1} f_{\alpha}, f_{\alpha}\right\rangle .
\end{aligned}
$$

Now we consider only the nonzero vectors $f_{\alpha}$. Clearly from the definition of direct sum, we have that only a countable number of them are nonzero. Thus renumbering them for convenience, we have

$$
\begin{aligned}
z & =\left\langle\left(A_{1} \otimes I_{2}\right) u, u\right\rangle=\sum_{\alpha \in \Lambda}\left\langle A_{1} f_{\alpha}, f_{\alpha}\right\rangle \\
& =\sum_{n=1}^{\infty}\left\langle A_{1} f_{n}, f_{n}\right\rangle=\sum_{n=1}^{\infty}\left\|f_{n}\right\|^{2} \frac{\left\langle A_{1} f_{n}, f_{n}\right\rangle}{\left\|f_{n}\right\|^{2}} \\
& =\sum_{n=1}^{\infty}\left\|f_{n}\right\|^{2}\left\langle A_{1} \psi_{n}, \psi_{n}\right\rangle,
\end{aligned}
$$


where $\psi_{n}=f_{n} /\left\|f_{n}\right\|$ with $\left\|\psi_{n}\right\|=1$. But $\left\langle A_{1} \psi_{n}, \psi_{n}\right\rangle$ is in $W\left(A_{1}\right)$ for each $n$, and $\sum_{n=1}^{\infty}\left\|f_{n}\right\|^{2}=1$. Therefore, $z$ is in $W\left(A_{1}\right)$ by the above mentioned theorem of Williams. Thus this proves that $W\left(A_{1} \otimes I_{2}\right)=W\left(A_{1}\right)$.

To complete the proof of the proposition, it is enough to show that $W\left(I_{3} \otimes A_{1}\right)=W\left(A_{1}\right)$. This follows readily from Proposition 3 and the proof given above.

In passing, we make the following remark. It is well known that the numerical range of a finite direct sum of operators is the convex hull of the numerical ranges of its summands [7, p. 113]. The proof of the generalization of this assertion to an infinite direct sum of operators follows easily from the theorem of Williams and the techniques used in the preceding proposition. Recall that $A_{1} \otimes I_{2}$ could alternatively be regarded as direct sum.

The following lemma is important in sequel. To proceed further, we refer the reader to the introduction for the definition of the operators $T_{j}$.

LEMma. The numerical range of the operator $T_{j}$ is the same as that of $A_{j}$ for each $j, 1 \leqq j \leqq n$; that is,

$$
W\left(T_{j}\right)=W\left(A_{j}\right), \text { for all } j, 1 \leqq j \leqq n .
$$

Proof. This follows readily from the repeated applications of Proposition 4 and the associative property of the tensor products.

Proof Of THEOREM. Clearly $W\left(T_{1}, \cdots, T_{n}\right) \subset \prod_{j=1}^{n} W\left(T_{j}\right)=\prod_{j=1}^{n} W\left(A_{j}\right)$. Consult the previous lemma.

Conversely, let $z=\left(z_{1}, \cdots, z_{n}\right)$ be an element of $\prod_{j=1}^{n} W\left(A_{j}\right)$. Then there exists $f_{j}$ in $\mathscr{H}_{j}$ with $\left\|f_{j}\right\|=1$ such that $z_{j}=\left\langle A_{j} f_{j}, f_{j}\right\rangle$ for all $j, 1 \leqq j \leqq n$. Then set $u=f_{1} \otimes \cdots \otimes f_{n}$. Thus $u$ is a unit vector in $\mathscr{H}_{1} \otimes \cdots \otimes \mathscr{H}_{n}$, and $z_{j}=\left\langle T_{j} u, u\right\rangle=\left\langle A_{j} f_{j}, f_{j}\right\rangle$, for all $j, 1 \leqq j \leqq n$. This implies that $z$ is in $W\left(T_{1}, \cdots, T_{n}\right)$. Thus $\prod_{j=1}^{n} W\left(A_{j}\right) \subset W\left(T_{1}, \cdots, T_{n}\right)$. This proves the theorem.

It is not known in general whether the joint spectrum of a commuting $n$-tuple of operators is contained in the closure of their joint numerical range [1]. However, by the remarks in the introduction we have the following

Corollary. $\quad \sigma\left(T_{1}, \cdots, T_{n}\right) \subset W\left(T_{1}, \cdots, T_{n}\right)$.

Proof.

$$
\begin{aligned}
\sigma\left(T_{1}, \cdots, T_{n}\right) & =\prod_{j=1}^{n} \sigma\left(A_{j}\right) \subseteq \prod_{j=1}^{n} \overline{W\left(A_{j}\right)} \\
& =\prod_{j=1}^{n} W\left(A_{j}\right)=\overline{W\left(T_{1}, \cdots, T_{n}\right)} .
\end{aligned}
$$


This also follows from above theorem and the fact that the joint spectrum of an $n$-tuple of commuting operators is contained in the closed convex hull of its joint numerical range [4].

Acknowledgement. I am grateful to Professor J. P. Williams for many helpful and informative conversations.

\section{REFERENCES}

1. A. T. Dash, Joint spectra, joint spectral sets and joint numerical range, Ph.D. Thesis, University of Toronto, 1969.

2. - Joint spectra, Studia Math. (to appear).

3. J. Dixmier, Les algèbres d'opérateurs dans l'espace Hilbertien, Gauthier-Villars, Paris, 1969.

4. N. Dekker, Ph.D. Thesis, Amsterdam, 1969.

5. A. T. Dash and M. Schechter, Tensor products and joint spectra, Israel J. Math. 8 (1970), 191-193. MR 41 \#5992.

6. A. Guichardet, Tensor products of $C^{*}$-algebras, Lecture Notes Series, nos. 12, 13, Aarhus Universitet, Mathematisk Institut, Denmark, 1969.

7. P. R. Halmos, A Hilbert space problem book, Van Nostrand, Princeton, N.J., 1967. MR 34 \#8178.

8. F. Treves, Topological vector spaces, distributions and kernels, Academic Press, New York, 1967. MR 37 \#726.

Department of Mathematics, Indiana University, Bloomington, Indiana 47401

Department of Mathematics, University of Guelph, Guelph, Ontario, Canada (Current address) 